\title{
Schwingungsverhalten von Langasit-Resonatoren bei unterschiedlicher Elektrodenleitfähigkeit
}

\author{
Schwerpunkt: A10
}

Silja Schmidtchen, Denny Richter, Holger Fritze, Institut für Energieforschung und Physikalische Technologien, TU Clausthal, Am Stollen 19B, 38640 Goslar 05321/6855-166, silja.schmidtchen@tu-clausthal.de

Piezoelektrische Resonatoren, die mit einer Metalloxidschicht versehen sind, können als Gassensoren genutzt werden, indem der Einfluss der elektrischen und mechanischen Eigenschaften dieser Schicht auf das Schwingungsverhalten betrachtet wird. Dabei können sowohl die Verschiebung der Resonanzfrequenz durch eine Änderung der Schichtmasse als auch die Veränderung von Amplitude bzw. Profil der Schwingung zur Messung herangezogen werden. Genutzt werden zwei verschiedene Elektrodengeometrien, die zum Mikrowaagemodus (vgl. Abbildung 1a) bzw. zum Leitfähigkeitsmodus (vgl. Abbildung 1b) führen. Mit Resonatoren, die im Leitfähigkeitsmodus betrieben werden, können Aussagen zum Sauerstoffpartialdruck getroffen werden. Hinzu kommen die im Mikrowaagemodus ermittelbaren Informationen über die Gaszusammensetzung, so dass die Kombination beider Messansätze zu einer erhöhten Selektivität der Sensoren führt.

Im Gegensatz zu herkömmlichen Quarz-Resonatoren, deren maximale Anwendungstemperatur bedingt durch eine starke Dämpfung bei ca. $450^{\circ} \mathrm{C}$ liegt, lassen sich Langasit-Resonatoren $\left(\mathrm{La}_{3} \mathrm{Ga}_{5} \mathrm{SiO}_{14}, \mathrm{LGS}\right)$ bis mindestens $1000^{\circ} \mathrm{C}$ als piezoelektrische Wandler einsetzen. Schwingungen lassen sich praktisch bis zur Schmelztemperatur von $1470^{\circ} \mathrm{C}$ anregen.

(a)

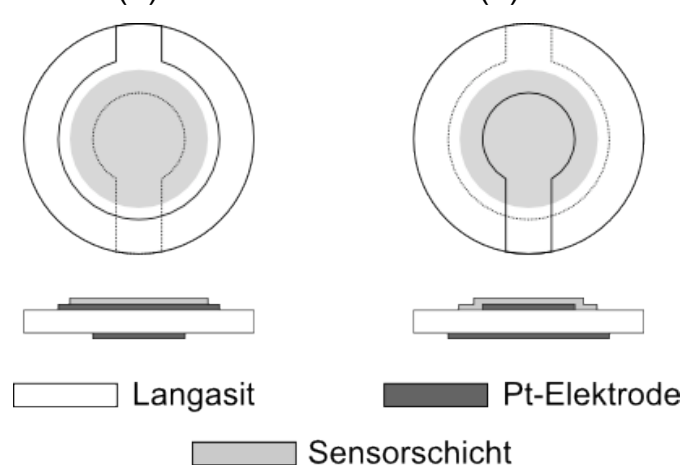

Abbildung 1: Schematische Darstellung des Elektroden- und Sensorschicht-Layouts für den Mikrowaage- (a) und Leitfähigkeitsmodus (b).

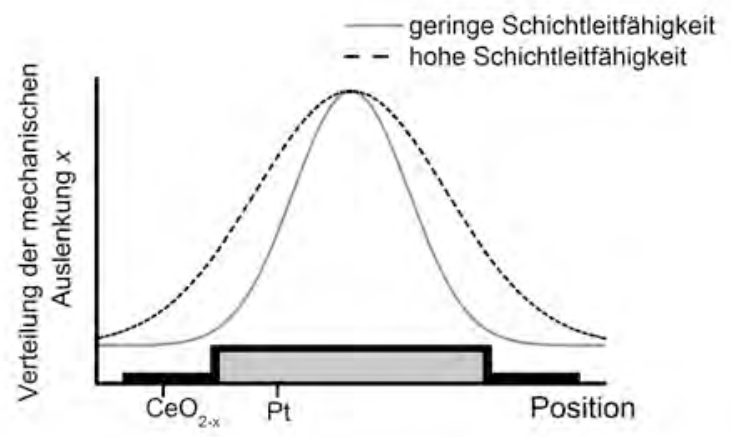

Abbildung 2: Veränderung der Leitfähigkeit einer Ceroxid-Schicht in Abhängigkeit vom Sauerstoffartialdruck

Unter Nutzung des bekannten Mikrowaageprinzips ${ }^{1}$ wird die Massenänderung der Schicht als Reaktion auf eine Veränderung in der umgebenden Atmosphäre in Form einer Resonanzfrequenzverschiebung gemessen. Im Leitfähigkeitsmodus wirkt sich die Veränderung der Zusammensetzung des Umgebungsgases auf die Leitfahigkeit der Schicht aus. Damit ist eine Änderung des Schwingungsprofils und der Resonanzfrequenz verbunden. Um den Messeffekt zu verstehen und zu optimieren, muss der Zusammenhang von Schichtleitfähigkeit und Verteilung der Schwingungsaplitude ermittelt werden. Zur Bestimmung der letztgenannten Größe wird ein Laser Doppler Interferometer verwendet.

Die Masseempfindlichkeit und das Schwingungsprofil eines Resonators werden hauptsächlich durch die Geometrien der Elektroden und der aufgebrachten Sensorschicht bestimmt. Die Schwingungsamplitude wie auch die Verteilung der Massensensivität folgen dabei annähernd einer Gauß-Verteilung ${ }^{2}$ mit einem Maximum in der Mitte der Elektrode. Durch das elektrische Feld zwischen den Elektroden wird dieser Bereich der y-orientierten Langasit-Resonatoren zu Scherschwingungen angeregt. Die Resonanzfrequenz wird durch die Dicke des Resonators inklusive Elektroden bestimmt. Außerhalb der Elektroden ist die Resonanzfrequenz durch das Fehlen der Elektroden höher, was eine Ausbreitung der Schwingung über die Elektroden hinaus einschränkt (Energy Trapping). 
Die Ausdehnung des Schwingungsprofils wird durch den Durchmesser der Elektroden beeinflusst. Um dies zu verifizieren werden Elektroden mit verschieden großen Durchmessern auf Vorder- und Rückseite des Resonators aufgebracht. Über der kleineren der beiden Elektroden wird zusätzlich eine Metalloxidschicht mit einem größerem Durchmesser als die Elektrode abgeschieden. Unter dem Einfluss reduzierender Atmosphären nimmt die Leitfähigkeit der Schicht zu, was in einem vergrößerten effektiven Elektrodendurchmesser resultiert (vgl. Abbildung 2).

Das Schwingungsprofil von Resonatoren mit verschiedenen Elektrodendurchmessern ist in der Folge dargestellt. Dazu werden mit dem Laser Doppler Interferometer die Schwingungsamplituden vom Grundton und Oberton bei Raumtemperatur im Line-Scan (Abbildung 3a) und Raster-Scan Verfahren ermittelt. Der Laser besitzt bei einem Arbeitsabstand von 0,5 m eine minimale Spotgröße von $18 \mu \mathrm{m}$ und erlaubt im Gegensatz zu anderen Verfahren zur Schwingungsprofilmessung eine (berührungslose) Messung auch bei hohen Temperaturen und verschiedenen Atmosphären. Es lassen sich lokale Auslenkungen bis in den nm-Bereich bestimmen ${ }^{3}$.

Als aktive Metalloxidschicht wird Ceroxid gewählt. Die Veränderung des Gauß-Profils der Schwingungsamplitude wird bei verschiedenen Sauerstoffpartialdrücken und Temperaturen bis $800{ }^{\circ} \mathrm{C}$ gemessen. Dabei ergibt sich eine Vergrößerung des effektiven Elektrodendurchmessers und somit die Verbreiterung des Schwingungsprofils (Abbildung 3b).

Weiterhin werden Messungen der Profile der Schwingungsamplituden an Resonatoren mit KeramikElektroden (Lanthan-Strontium-Manganit, LSM) durchgeführt. Bei hohen Temperaturen werden diese Elektroden leitfähig, so dass sich auch das Schwingungsprofil der entsprechenden Resonatoren mit der Temperatur ändert.

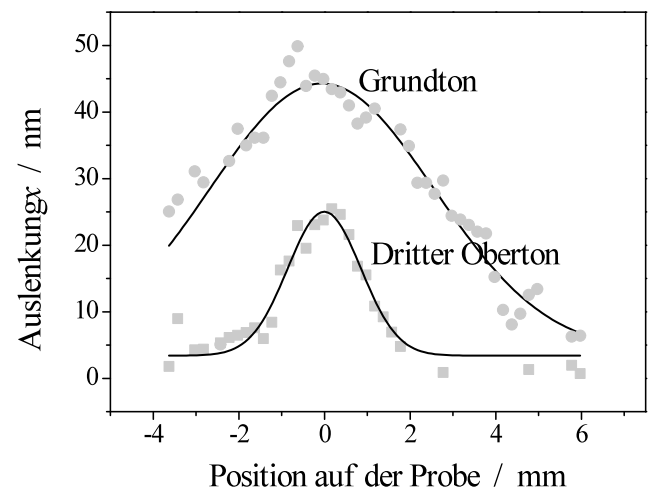

(a)

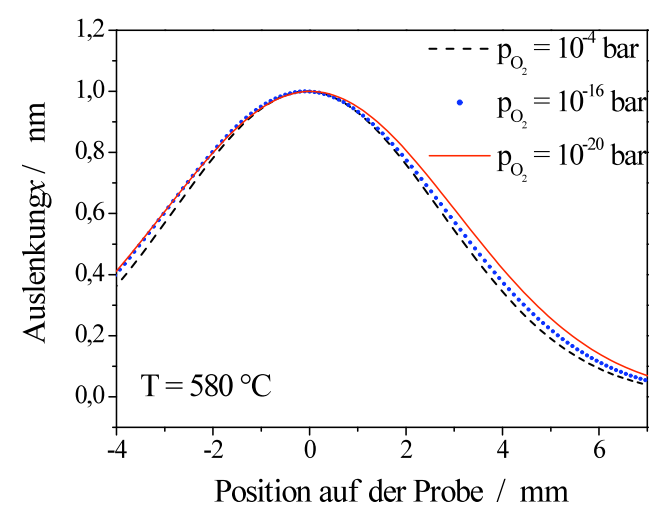

(b)

Abbildung 3: (a) Räumliche Verteilung der Auslenkung eines Resonators, angeregt mit den Resonanzfrequenzen des Grundtons (ca. $2 \mathrm{MHz}$ ) und eines Obertons (ca. $6 \mathrm{MHz}$ ) bei Raumtemperatur. Anregungsspannung $\mathrm{U}_{\mathrm{A}}=2 \mathrm{~V}$ für den Grundton und $5 \mathrm{~V}$ für den Oberton. (b) Schwingungsprofil eines Resonators mit $\mathrm{CeO}_{2-\mathrm{x}}$-Schicht bei $580{ }^{\circ} \mathrm{C}$ und drei verschiedenen Sauerstoffpartialdrücken.

\footnotetext{
${ }^{1}$ Sauerbrey, G. (1959), 'Verwendung von Schwingquarzen zur Wägung dünner Schichten und zur Mikrowägung', Zeitschrift für Physik A Hadrons and Nuclei 155, 206-222.

${ }^{2}$ Martin, B. A. und Hager, H. E. (1989), 'Velocity profile on quartz crystals oscillating in liquids', J. Appl. Phys. 65(7), 2630-2635.

${ }^{3}$ Schmidtchen, S.; Sauerwald, J.; Xia, H. und Fritze, H. (2010), 'Displacement Characteristics of Piezoelectric Langasite Transducers at High Temperatures', AIP Conference Proceedings 1253(1), 164-170.
} 